\title{
An Algorithm for Neuropathic Pain Management in Older People
}

\author{
Gisèle Pickering ${ }^{1,2,3} \cdot$ Margaux Marcoux $^{1} \cdot$ Sylvie Chapiro $^{4} \cdot$ Laurence David $^{5} \cdot$ \\ Patrice Rat $^{6} \cdot$ Micheline Michel $^{7} \cdot$ Isabelle Bertrand $^{8} \cdot$ Marion Voute $^{1}$. \\ Bernard Wary ${ }^{9}$
}

Published online: 10 August 2016

(c) The Author(s) 2016. This article is published with open access at Springerlink.com

\begin{abstract}
Neuropathic pain frequently affects older people, who generally also have several comorbidities. Elderly patients are often poly-medicated, which increases the risk of drug-drug interactions. These patients, especially those with cognitive problems, may also have restricted communication skills, making pain evaluation difficult and pain treatment challenging. Clinicians and other healthcare providers need a decisional algorithm to optimize the recognition and management of neuropathic pain. We present a decisional algorithm developed by a multidisciplinary group of experts, which focuses on pain assessment and therapeutic options for the management of neuropathic pain,
\end{abstract}

Gisèle Pickering

gisele.pickering@udamail.fr

1 Centre de Pharmacologie Clinique, Bâtiment 3C, CHU

Clermont-Ferrand, 58 rue de Montalembert, 63001

Clermont-Ferrand Cedex, France

2 Inserm, CIC 1405, UMR Neurodol 1407, 63003

Clermont-Ferrand, France

3 Laboratoire de Pharmacologie, Faculté de Médecine, Université de Clermont, 63001 Clermont-Ferrand, France

4 Palliative Care, Hôpital Paul Brousse, 94800 Villejuif, France

5 CETD, Centre Hospitalier de la Côte Basque, 64100 Bayonne, France

6 Department of Geriatrics, Marseille University Hospital, 13000 Marseille, France

7 Department of Geriatrics, Rennes University Hospital, 35000 Rennes, France

8 Sanofi Pasteur MSD, 162 Avenue Jean Jaurès, 69367 Lyon Cedex 07, France

9 Palliative Care, Hôpital de Metz, France Service Régional De Soins Palliatifs, Hôpital Beauregard, CHR Metz-Thionville, 57126 Thionville, France particularly in the elderly. The algorithm involves four main steps: (1) detection, (2) evaluation, (3) treatment, and (4) reevaluation. The detection of neuropathic pain is an essential step in ensuring successful management. The extent of the impact of the neuropathic pain is then assessed, generally with self-report scales, except in patients with communication difficulties who can be assessed using behavioral scales. The management of neuropathic pain frequently requires combination treatments, and recommended treatments should be prescribed with caution in these elderly patients, taking into consideration their comorbidities and potential drug-drug interactions and adverse events. This algorithm can be used in the management of neuropathic pain in the elderly to ensure timely and adequate treatment by a multidisciplinary team.

\section{Key Points}

Neuropathic pain can be difficult to diagnose in elderly people, particularly when they have communication difficulties.

It is important that patients with suspected neuropathic pain are assessed and treated in a timely fashion by the multidisciplinary team involved in their healthcare.

The treatment of neuropathic pain in the elderly is complex, and treatment failure is frequent.

The four-step algorithm presented here can be adapted for use in the management of elderly people with neuropathic pain.

The four steps of the algorithm are detection and evaluation of pain, treatment, and re-evaluation. 


\section{Background}

The increasing life expectancy in many countries is leading to an increasingly older population; since chronic pain is frequent in older people, its management in this population is increasingly relevant. Neuropathic pain (NP) is "chronic pain arising as a direct consequence of a lesion or disease affecting the somatosensory system' [1]. It is particularly complex and difficult to treat and has a high treatmentfailure rate $[2,3]$. Published estimates for pain prevalence range from $3 \%$ using the Berger criteria to $9.8 \%$ using clinical examination (gold standard) in the community depending on the methods and the population [4, 5]. Results from a postal survey in France showed a prevalence of $6.9 \%$, and surveys in the UK and France reported a prevalence of 7-8\% for chronic pain with neuropathic characteristics in the general population $[6,7]$. Using the self-report version of the Leeds Assessment of Neuropathic Symptoms and Signs pain scale (S-LANSS), a prevalence of $8.8 \%$ was reported in the UK $[8,9]$. The prevalence was reported to be $9.3 \%$ in individuals aged 50-64 years in the general population in the UK and France [7]. However, only sparse data are available for the prevalence in older and very old people. Most methods used to obtain estimates rely on patients being able to communicate and are not adapted for older people with cognitive impairment or communication difficulties.

The risk of cognitive impairment and dementia rises with age and increases difficulties with communication. Cognitive impairment has been reported to be present in up to $68 \%$ of the elderly in long-term care facilities [10]. Dementia is one of the leading causes of disability and dependence among the elderly, affecting $>35$ million people worldwide, $3-11 \%$ of those aged $>65$ years, and $20-50 \%$ of those aged $>85$ years $[11,12]$. Little research has been conducted to estimate the prevalence of pain in patients with dementia and other neurodegenerative diseases (e.g., stroke, Parkinson's disease) or the relationship between pain and the different subtypes of dementia $[13,14]$.

The care of older patients with cognitive impairment, communication disabilities, or dementia is a real challenge because these disorders make the assessment and the treatment of chronic pain difficult. Self-reporting is considered to be the gold standard for pain evaluation, but older patients with these pathologies are often unable to communicate verbally, and tools for nonverbal communication must be used [15]. Comorbidities and polypharmacy, which are more common with advancing age, increase the risk of iatrogenic disease and serious drugdrug interactions because of the physiological and pharmacological changes associated with aging $[2,3,16]$.
Chronic pain, such as that associated with osteoarthritis, is very common in the elderly and can cause sleep difficulties, leading to a compromised quality of life [17]. NP is often more debilitating than other chronic pain because of the extremely painful paroxysms, such as stabbing, burning, electric shock, allodynia, hyperalgesia; when present in addition to other chronic pain, NP can further affect patients' quality of life. NP is frequently resistant to available pain treatment, but it is essential to optimize its management in the elderly, especially those with cognitive impairment [18]. Current recommendations on pain assessment and management give general guidance to the management of NP. In current practice, there is a specific need to build on published guidelines for focusing on NP for elderly patients, especially for those with cognitive impairment $[15,19]$. The aim of this paper is to describe a decisional algorithm with specific recommendations to improve the overall management of NP in the elderly, particularly those with communication difficulties. The algorithm has four components that cover the detection and diagnosis, evaluation, treatment, and re-evaluation of NP, with key points related to geriatric patients.

\section{Algorithm Development}

\subsection{Algorithm Development Team}

A group of medical experts comprising geriatricians, palliative care doctors, pharmacologists, pain clinic practitioners, and palliative care nurses participated in the algorithm development team that designed a decisional algorithm for NP detection and diagnosis, evaluation, treatment, and re-evaluation. To be eligible, the participants had to have between 10 and 30 years of experience in clinical care for patients with pain and/or elderly patients with different degrees of cognitive impairment. These participants formed the Doloplus ${ }^{\circledR}$ group team, who have validated and translated two behavioral scales, Doloplus ${ }^{\circledR}$ and Algoplus ${ }^{\circledR}$, for pain evaluation in older patients with limited ability to communicate [20-22].

\subsection{Algorithm Development Process}

The group searched PubMed for publications on tools used for NP assessment and for general guidelines on pain management in older individuals. The members of the group discussed their experiences, the limitations of NP management in cognitively impaired patients and held regular face-to-face meetings over 1 year, during which the algorithm was developed taking into account recommendations from geriatric and pain societies. After the 
algorithm was designed, it was sent to 50 members of the French Pain Society (SFETD) and the French Geriatrics Society for evaluation and feedback; it was also presented at the SFETD and at the Neuropathic Pain Special Interest Group (NeuPSIG) congresses in 2015. Feedback received was assessed and used by the Doloplus group team to validate the final version of the algorithm. We present here the final, consensual version of the algorithm.

\section{Algorithm}

The validated algorithm comprises four steps:

1. Detection and diagnosis of NP: pathological context and clinical signs.

2. Evaluation of NP and its impact: auto-evaluation and assessment with behavioral tools.

3. General guidelines for NP management and treatment approaches.

4. Re-evaluation of pain and treatment.

\subsection{Step 1: Detection and Diagnosis of Neuropathic Pain (NP)}

Older adults may underreport pain or have difficulties communicating about pain, even in the absence of cognitive impairment; therefore, physicians may undertreat pain [23]. It is important to remember that NP can be hidden by nociceptive pain, and healthcare practitioners must observe patients, looking for any warning signs of the presence of chronic pain with neuropathic characteristics.

Any change of behavior in elderly patients, especially those with communication disorders impacting pain expression, should lead the physician to suspect the presence of pain. The patient's file must be checked to identify any pathology in their past history that could potentially lead to NP (Fig. 1) [17, 24]. Incorporating surrogate reporting, such as asking the patient's family about the patient's expression of pain, behavioral disorders, and their past history, can provide valuable information that may be missing from the patient's file.

Observation and clinical examination are at the heart of NP diagnosis. Protecting a particular part of their body, adopting an analgesic position in bed, paroxysmal bouts of shouting or sighing, or apathetic behavior can all suggest breakthrough pain or NP features (e.g., burning, tingling, electric shocks) that the patient may be unable to describe and discriminate. The physician should look for clinical signs that potentially lead to NP (cutaneous scarring, skin abnormality) and self-protection. Clinical examination should focus especially on the presence of two prominent symptoms: allodynia (a painful response to a normally innocuous stimulus) and hyperalgesia (an increased pain response to a normally painful stimulus) [25]. These symptoms often occur when the patient is being washed or during mobilization. Confirmation can be obtained by gentle stimulation with cotton wool and finger pressure on the suspected zone. Relief or degradation of the behavioral discomfort can be detected with stimulation, massage, or the application of hot or cold stimuli. Attempts should be made to identify the etiology of the elicited signs and symptoms.

\subsection{Evaluation of NP and its Impact}

Self-report is the gold standard for assessing pain, but information may be difficult to obtain, even if the patient has no cognitive impairment [15]. Older patients may estimate that pain is normal with aging or hesitate to complain because they are afraid to lose their independence or to take additional medical tests and interventions [26]. In the elderly, pain can be evaluated with a numerical or verbal rating scale (NRS or VRS); visual analog scales (VAS) are less recommended for this age group. Elderly patients with mild to moderate dementia may still be able to provide a reliable report of pain, even if self-report is not always possible. Comparisons and metaphors may help patients characterize their pain, and open-ended questions without follow-up questions are recommended [15]. More than 30 scales are available for elderly patients with cognitive impairment [13, 27]. These tools can determine whether the patient suffers from pain but cannot determine whether the pain is neuropathic. Despite variable content across tools, items to identify pain or discomfort overlap considerably [28]. Specific scales exist for NP, but none of them are adapted for patients with communication disorders because they all require patient participation (Table 1) [8, 9, 29-35]. Evaluation of quality of life and functional status, including cognitive psychological and depression/ anxiety questionnaires, are also part of the global evaluation of geriatric patients (Fig. 2).

If self-evaluation is possible, an NP scale may be used in the elderly following usual instructions for use of the scale (Table 1). If the NP scale gives a negative result whereas previous evaluation had shown pain and NP was suspected, the cause could be nociceptive rather than neuropathic. Treatment of nociceptive pain should then be attempted, with repeated reassessment.

Among the behavioral scales, Doloplus-one of the first published scales - and Algoplus have been validated for pain evaluation in elderly patients with communication disorders [21, 22]. Doloplus has three dimensions (somatic, psychomotor, and psychosocial reactions) each comprising ten items (rated from 0 to 3). Algoplus focuses on acute pain evaluation and has five items (facial expression, look, 


\section{WARNING SIGNS for the presence of neuropathic pain (NP)}

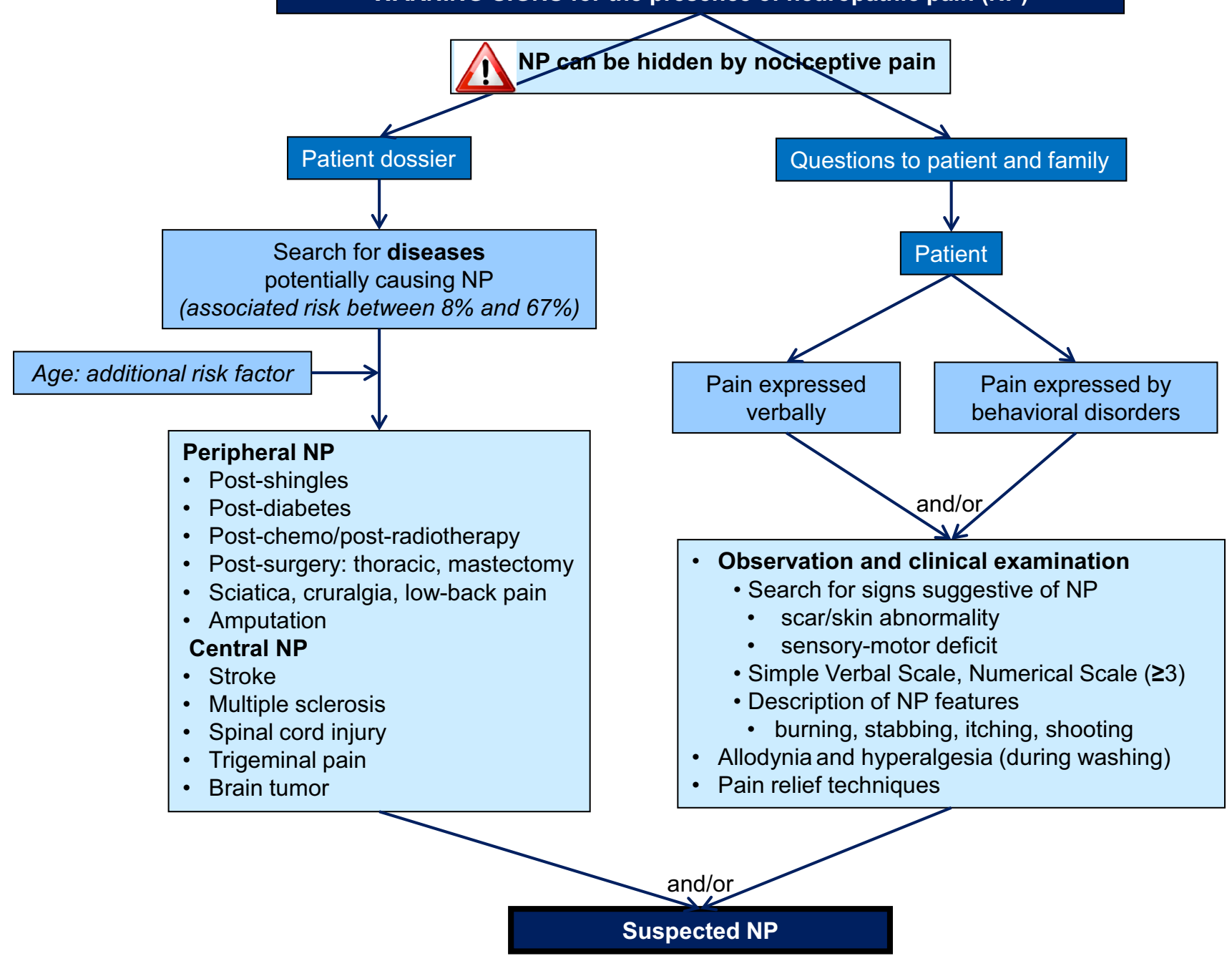

Fig.1 Algorithm part 1: detection and diagnosis of neuropathic pain

Table 1 Tools for assessing neuropathic pain

\begin{tabular}{|c|c|}
\hline Tool name & Brief description \\
\hline IDPain [34] & $\begin{array}{l}\text { Five items 'yes' score }=1 \text {; sixth item 'yes' score }=-1 \text {; all items 'no' score }=0 \text {, results displayed } \\
\text { as a diagram to situate pain }\end{array}$ \\
\hline $\begin{array}{l}\text { Leeds Assessment of Neuropathic Symptoms } \\
\text { and Signs (LANSS) [8] }\end{array}$ & $\begin{array}{l}\text { LANSS: } 5 \text { items on neuropathic symptoms; } 2 \text { items on clinical examination } \\
\text { S-LANSS: } 7 \text { items rated as present or absent; } 2 \text { items to evaluate pain distribution and intensity }\end{array}$ \\
\hline \multicolumn{2}{|l|}{ Self-report LANSS (S-LANSS) [9] } \\
\hline Neuropathic Pain in 4 questions (NP4) [29] & Four questions divided into 10 items: 'yes' score $=1$; 'no' score $=0$ \\
\hline Neuropathic Pain Questionnaire (NPQ) [33] & Total 12 items: 10 items related to sensory or sensation responses; 2 items related to affect $^{\mathrm{a}}$ \\
\hline The Neuropathic Pain Scale (NPS) [32] & $\begin{array}{l}\text { Total } 10 \text { items: } 7 \text { items on pain characteristics (intensity, sharpness, hotness, dullness, coldness, } \\
\text { skin sensitivity, and itchiness); } 1 \text { item on time when pain is present; } 1 \text { item on overall } \\
\text { unpleasantness; } 1 \text { item on the intensity of deep and surface pain }\end{array}$ \\
\hline $\begin{array}{l}\text { Neuropathic Pain Symptom Inventory (NPSI) } \\
\text { [30] }\end{array}$ & $\begin{array}{l}\text { Five parts (spontaneous burning pain, spontaneous deep pain, paroxysmal pain, evoked pain, } \\
\text { and paresthesia/dysesthesia) divided into } 10 \text { pain descriptors }\end{array}$ \\
\hline painDETECT [31] & $\begin{array}{l}\text { Nine items: } 7 \text { weighted sensory descriptor items and } 2 \text { items relating to the spatial and temporal } \\
\text { characteristics of the individual pain pattern }\end{array}$ \\
\hline
\end{tabular}

${ }^{a}$ NPQ Short-form comprising three items: numbness, tingling, and pain increase in response to touch [35] 
Systematic evaluation for suspected neuropathic pain (NP)

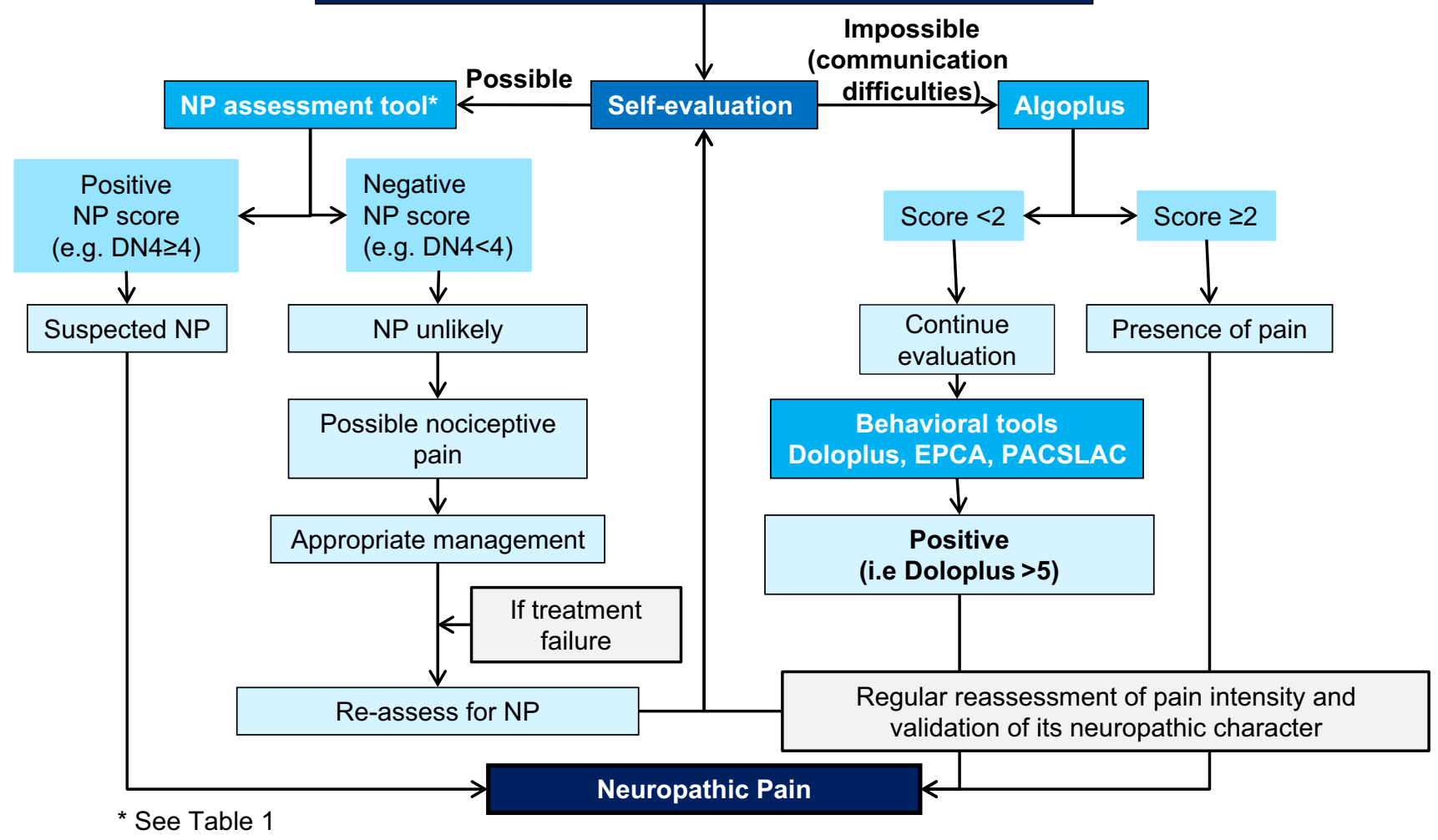

Fig. 2 Algorithm part 2: evaluation of neuropathic pain. DN4 'Douleur Neuropathique 4' pain questionnaire, EPCA Echelle Comportementale pour Personnes Âgées (Behavioral Scale for Elderly

complaints, body position, atypical behavior): each item is scored 'yes' $=1$ or 'no' $=0$. A score of $\geq 2$ for Algoplus and $\geq 5$ for Doloplus indicates the presence of pain. In the algorithm, the Doloplus and Algoplus tools were combined to give a NP scale.

If self-evaluation is not possible and NP is suspected, we propose to start evaluation with the Algoplus scale. An Algoplus score $\geq 2$ indicates pain is present and should be treated. Treatment should be attempted and the pain regularly reassessed with re-evaluation of the neuropathic character of pain. A score of $<2$ with the Algoplus scales does not mean the patient has no pain. A recent study showed that $17 \%$ of patients with a Algoplus score $<2$ had a positive Doloplus score. Therefore, we recommend that patients with an Algoplus score $<2$ should be evaluated with Doloplus or another behavioral scale (e.g. Echelle Comportementale pour Personnes Âgées [Behavioral Scale for Elderly Persons: ECPA] or Pain Assessment Checklist for Seniors with Limited Ability to Communicate [PACSLAC]). A score of $\geq 5$ with the Doloplus scale indicates pain is present and the patient should be treated, with further evaluation and reassessment.
Persons), PACSLAC Pain Assessment Checklist for Seniors with Limited Ability to Communicate

\subsection{General Guidelines for NP Management and Treatment Approaches}

Effective pain management in elderly patients begins with a comprehensive and accurate evaluation to be able to select the most appropriate treatment strategy, including pharmacological and non-pharmacological approaches [19]. Treatment efficacy and adverse events should then be evaluated and reevaluated. Pharmacological treatment is usually the first-line treatment for NP, but non-pharmacological approaches should be added when possible. NP treatment guidelines in the elderly are generally based on international recommendations [19]. Drug treatments have been reported to have limited efficacy in the elderly, but data and studies specifically for elderly patients are lacking. NP should be tackled within the specific setting of the geriatric patient, and the choice of medication will depend on their clinical situation, comorbidities, renal function, other medications, and cognitive status (Fig. 3).

The elderly may have previous serious illnesses accompanied by significant pain and symptom burden. Comorbidities associated with polypharmacy may complicate the evaluation, management, and treatment of NP, 
Treatment for neuropathic pain (NP)
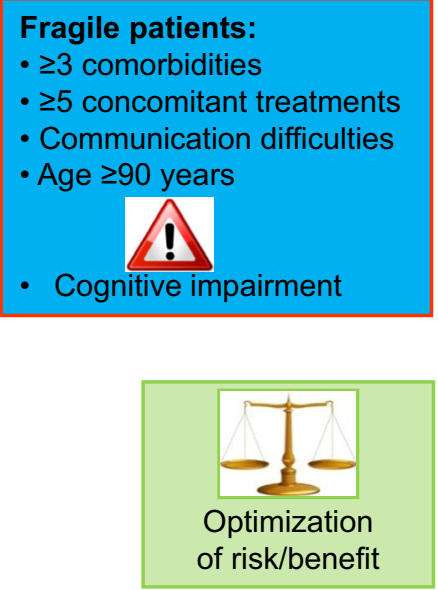

Renal function assessment

Take into consideration health status

Anticipation adverse events

Favor drugs with fewer drug-drug-interactions

- Verify treatment adherence

- Surveillance for adverse effects

- Continue treatment for several weeks since onset of action sometimes long

- if inefficient but well-tolerated increase dose up to highest, authorized or tolerated dose

- Evaluate and reevaluate the efficacy (pain scale) as well as the impact on the quality of life

Fig. 3 Algorithm part 3: treatment of neuropathic pain

and patients are more likely to experience adverse effects $[23,36]$. It has been reported that $31 \%$ of patients aged $\geq 85$ years present four or more chronic comorbidities [15]. Polypharmacy is frequent, and it has been reported that two-thirds of community-dwelling people aged $>60$ years take four or more medications daily; frail, elderly people with multiple comorbidities take an average of nine; and elderly hospital inpatients may take as many as 14 [15]. Non-prescription drugs, especially paracetamol, should be taken into consideration in community-dwelling patients. A number of physiological changes that can affect drug metabolism are also associated with aging, especially diminution of renal function [2, 23, 37, 38]. Adverse events such as constipation, vomiting, or nausea should be anticipated in older patients, especially in those using opioids $[19,39]$. Drugs with the least potential drug-drug interactions should be preferred [39]. In older patients, the 'start low' and 'go slow' approach should be used, i.e., start treatment at the lowest dose and titrate up depending on efficacy and tolerability. Monotherapy, local treatments, and oral rather than intravenous routes should be preferred $[37,40]$.

Optimization of the benefit/risk balance is important, so frequent monitoring for overdose and efficacy should be planned. Treatment adherence should be verified even in patients who have no or only mild cognitive impairment. Beliefs about the treatment and its cost, efficacy, or adverse effects may lead to non-adherence in $40-75 \%$ of older patients, who may take more than the prescribed dose, forget to take, or discontinue the treatment [26, 41]. Patients should be informed that the onset of action with antidepressants for NP treatment may take up to 2 weeks and may necessitate higher doses, and therefore they should continue the treatment for several weeks. The objectives of pain treatment should be explained to the patients, and they should understand that the goal of zero pain will rarely be reached. Reassessment of pain should include an evaluation of its impact on quality of life using, for example, the Patient Global Impression of Change (PGIC) [42]. If the patient is cognitively impaired, the clinical team will need to reevaluate with behavioral scales, observe all the above points, and monitor adverse events.

Pain may be difficult to diagnose in patients who are agitated and who present behavioral or psychological symptoms of dementia (BPSD); however, drugs for BPSD (including antipsychotics, anticonvulsants, antidepressants, anxiolytics, cholinesterase inhibitors, and $N$-methyl-D-aspartate-receptor modulators) should only be used after pain has been eliminated as a cause of the symptoms and after non-pharmacological treatments have been tried [19].

The pharmacological treatment of NP has been well described, but special considerations are needed in elderly patients, especially those with cognitive impairment, as they have generally been excluded or under-represented in clinical trials [19, 43, 44]. First-line treatment for NP includes antidepressants, anti-epileptics, lidocaine $5 \%$, and 
capsaicin plasters, but treatment availability and prescription authorizations vary between countries. The efficacy of tricyclic antidepressants (TCAs) (amitriptyline, imipramine, clomipramine) for NP has been well established with a number needed to treat (NNT) of 3.6 and number needed to harm (NNH) of 13.4 [44]. However, the anticholinergic effects of TCAs (visual, urinary, gastrointestinal) mean they must be used with caution in older patients as well as in those with cardiovascular and cardiac diseases. Antidepressants also have many drug-drug interactions that need to be monitored. Gabapentinoids are also first-line treatments (NNT 7.7, NNH 13.9) that have a better safety profile, with minimal concern for drug-drug interactions and no interference with hepatic enzymes; however, they do induce central adverse events, including sedation, ataxia, or edema [38]. Although lidocaine plasters have been shown to result in less cognitive impairment than have antidepressants in older patients with post-herpetic neuralgia (PHN), a recent publication suggests the quality of the available evidence is too low for them to be considered as a first-line treatment option [17, 43]. Other serotonin-norepinephrine reuptake inhibitor (SNRI) antidepressants (e.g., venlafaxine, duloxetine, milnacipran) have a better safety profile than TCAs (NNT 6.4, NNH 11.8) [43]. However, these drugs have the potential for adverse events such as nausea, constipation, hot flashes, hyperhidrosis, palpitations, dry mouth, hypertension, and drug-drug interactions as well as a risk of serotonergic syndrome and therefore must be used very cautiously in the elderly.

Tramadol is an effective second-line analgesic for NP (NNT 4.7, NNH 12.6) but can give rise to adverse events such as nausea, constipation, drowsiness, and seizures and may increase cognitive impairment [43]. It should not be combined with antidepressants, commonly prescribed in the elderly, because of the risk of serotonergic syndrome, which is rarely lethal but increases confusion and general ill health in elderly patients. Capsaicin patches are effective for second-line treatment of peripheral NP, such as PHN, but the burning sensation and stinging may be intolerable, and data regarding their use in elderly patients are few $[19,45,46]$. However, a recent study that included patients up to the age of 80 years found the capsaicin patch offered a faster onset of pain relief and an overall higher level of satisfaction than did pregabalin [47].

Strong opioids (morphine, oxycodone, methadone, tapentadol) have been shown to be efficacious in the treatment of peripheral NP (NNT 4.3, NNH 11.7) and are no longer considered as only end-of-life drugs. Some of their side effects, such as constipation, nausea, or vomiting, should be monitored and treated in elderly patients, especially those with cognitive impairment. Sedation, cognitive impairment, respiratory depression, and psychomotor impairment can be managed with an approach that includes slow titration and adequate hydration of the patient. These drugs are currently considered a third-line treatment

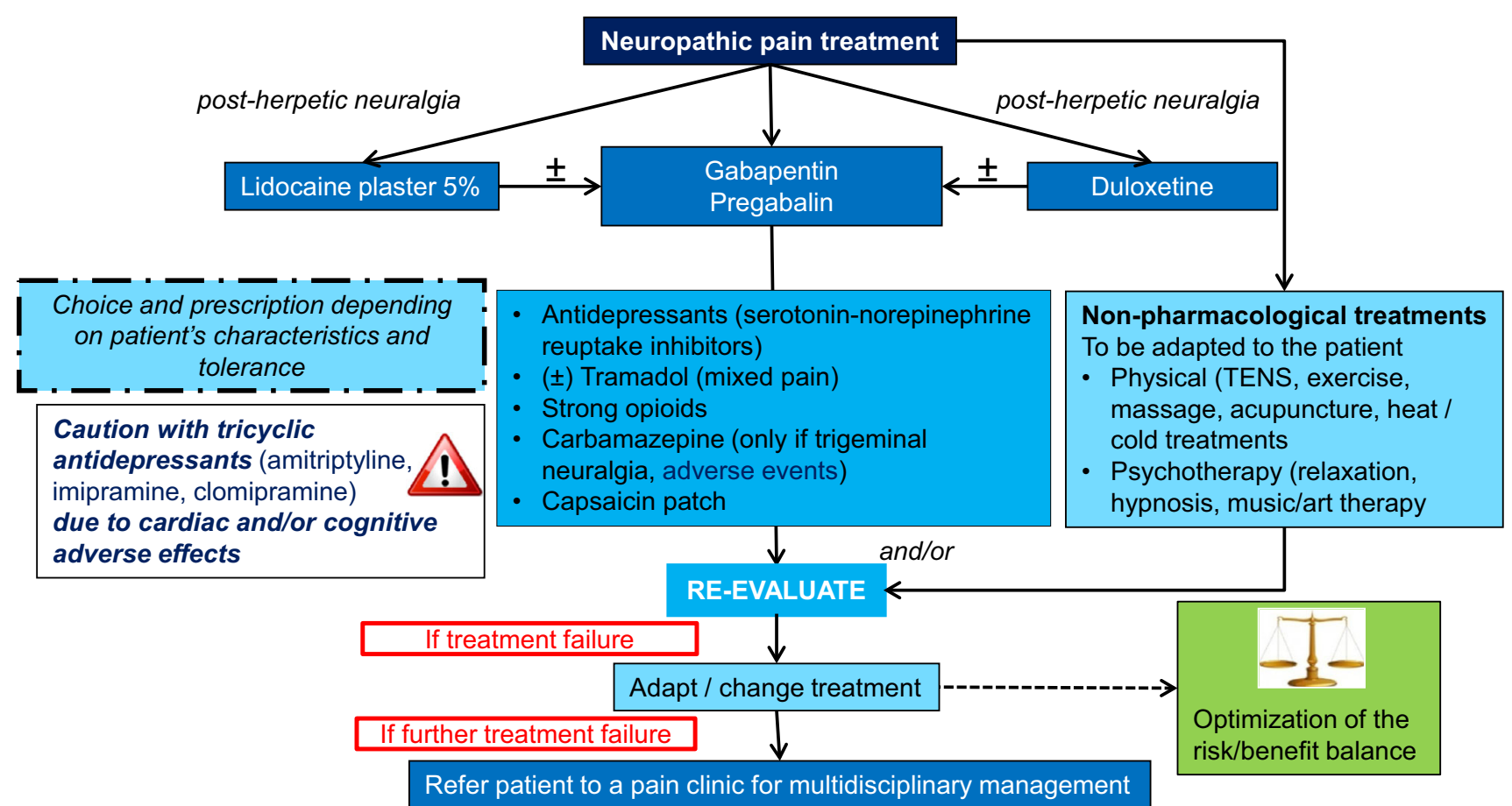

Fig. 4 Algorithm part 4: treatment and re-evaluation of neuropathic pain. TENS transcutaneous electrical nerve stimulation 
because of the potential risk of abuse, a higher risk of mortality due to prescription-related overdose, diversion, misuse, and morbidity [43]. However, these considerations may not be relevant for elderly patients with NP and communication difficulties in long-term care facilities. Other anti-epileptics, such as carbamazepine and sodium valproate, that can be used for third-line NP treatment have many side effects and drug-drug interactions and should, therefore, be used cautiously in the elderly.

In the elderly, as for any patient with pain, if a combination of drugs is required, they should have different mechanisms of action. However, this approach should be used only when absolutely necessary and the drugs should be hierarchized to reduce the drug load and the risk of drugdrug interactions. A multimodal approach is recommended that includes both pharmacological and non-pharmacological treatments based on available evidence [40]. The role of non-pharmacological treatments for managing pain in the elderly is becoming increasingly important because they are safe, may relieve pain, and can enhance the effects of pharmacological treatment [45]. Additionally, many nonpharmacologic treatments used for patients with cognitive impairment involve behavioral techniques and exercise and have been reported to reduce pain and, in some cases, result in improved functioning [39]. However, longer-term studies in larger elderly populations are needed.

\subsection{Re-evaluation of NP}

The efficacy of pain treatment in elderly patients with communication and cognition disorders must be regularly reevaluated by pain assessment in collaboration with all the people involved in caring for the patient. An important goal is to minimize adverse events from drug treatments, and thus improve the benefit-risk ratio. The evaluations should be repeated frequently and discussed by the multidisciplinary healthcare team, who can decide what changes in the therapeutic strategy, if any, are required (Fig. 4).

The present algorithm could be a useful practical tool in everyday practice. Its main limitation is that it has not been validated in real life. The Doloplus group is planning to validate the four steps of the algorithm in a prospective study involving a large population of older people with cognition impairment in the near future. This validation will enable the tool to be widely disseminated to healthcare providers who work with geriatric populations.

\section{Conclusions}

NP is a very challenging issue in elderly patients, particularly those with communication disorders or restricted communication skills. Every step is important, and evaluation should be a natural reaction for healthcare providers for patients with behavioral changes. Many tools are available, and they must be used adequately. The algorithm presented here could be a useful tool for clinical investigation in the elderly with cognitive impairment. Pharmacological treatments should be prescribed cautiously because, although the pharmacology of a drug may be known when used alone, it may be altered when used with other drugs and in elderly patients. Non-pharmacological treatments can be considered, but evidence for their efficacy is limited. Frequent re-evaluation and multidisciplinary team collaboration are important elements in providing the best quality of care for these patients with suspected NP. The algorithm presented here can be adapted and used to ensure that cognitively impaired elderly patients with suspected NP can be diagnosed and managed efficiently and efficaciously.

\section{Compliance with Ethical Standards}

Conflict of interest IB is employed by Sanofi Pasteur MSD who funded editorial assistance. GP, MMa, SC, LD, PR, MMi, MV and BW have no conflicts of interest.

Funding No funding was used to support the work described in this manuscript. All authors acknowledge editorial assistance from Margaret Haugh (MediCom Consult, Villeurbanne, France), funded by Sanofi Pasteur MSD.

Open Access This article is distributed under the terms of the Creative Commons Attribution-NonCommercial 4.0 International License (http://creativecommons.org/licenses/by-nc/4.0/), which permits any noncommercial use, distribution, and reproduction in any medium, provided you give appropriate credit to the original author(s) and the source, provide a link to the Creative Commons license, and indicate if changes were made.

\section{References}

1. Treede RD, Jensen TS, Campbell JN, Cruccu G, Dostrovsky JO, Griffin JW, et al. Neuropathic pain: redefinition and a grading system for clinical and research purposes. Neurology. 2008;70(18):1630-5.

2. Lussier D, Pickering G. Pharmacological considerations in older patients. In: Beaulieu P, Lussier D, Porreca F, Dickenson A, editors. Pharmacology of pain. Seattle: ISAP Press; 2010.

3. Pickering G, Capriz-Ribiere F. Neuropathic pain in the elderly. Psychol Neuropsychiatr Vieil. 2008;6(2):107-14.

4. Berger A, Dukes EM, Oster G. Clinical characteristics and economic costs of patients with painful neuropathic disorders. J Pain. 2004;5(3):143-9.

5. Yawn BP, Wollan PC, Weingarten TN, Watson JC, Hooten WM, Melton LJ 3rd. The prevalence of neuropathic pain: clinical evaluation compared with screening tools in a community population. Pain Med. 2009;10(3):586-93.

6. Bouhassira D, Lanteri-Minet M, Attal N, Laurent B, Touboul C. Prevalence of chronic pain with neuropathic characteristics in the general population. Pain. 2008;136(3):380-7. 
7. Fine PG. Chronic pain management in older adults: special considerations. J Pain Symptom Manage. 2009;38(2 Suppl):S4-14.

8. Bennett M. The LANSS Pain Scale: the Leeds assessment of neuropathic symptoms and signs. Pain. 2001;92(1-2):147-57.

9. Bennett MI, Smith BH, Torrance N, Potter J. The S-LANSS score for identifying pain of predominantly neuropathic origin: validation for use in clinical and postal research. J Pain. 2005;6(3):149-58.

10. Alzheimer's Association. 2010 Alzheimer's disease facts and figures. Alzheimer Dis Assoc Disord. 2010;6(2):158-94.

11. Corbett A, Husebo BS, Achterberg WP, Aarsland D, Erdal A, Flo E. The importance of pain management in older people with dementia. Br Med Bull. 2014;111(1):139-48.

12. Dziechciaz M, Balicka-Adamik L, Filip R. The problem of pain in old age. Ann Agric Environ Med. 2013;Spec No. 1:35-8.

13. Defrin R, Amanzio M, de Tommaso M, Dimova V, Filipovic S, Finn DP, et al. Experimental pain processing in individuals with cognitive impairment: current state of the science. Pain. 2015;156(8):1396-408.

14. van Kooten J, Delwel S, Binnekade TT, Smalbrugge M, van der Wouden JC, Perez RS, et al. Pain in dementia: prevalence and associated factors: protocol of a multidisciplinary study. BMC Geriatr. 2015;15:29.

15. Herr K. Pain assessment strategies in older patients. J Pain. 2011;12(3 Suppl 1):S3-13.

16. Pickering G, Pereira B, Clere F, Sorel M, de Montgazon G, Navez M, et al. Cognitive function in older patients with postherpetic neuralgia. Pain Pract. 2014;14(1):E1-7.

17. Pickering ME, Chapurlat R, Kocher L, Peter-Derex L. Sleep disturbances and osteoarthritis. Pain Pract. 2016;16(2):237-44.

18. Hansson PT, Attal N, Baron R, Cruccu G. Toward a definition of pharmacoresistant neuropathic pain. Eur $\mathrm{J}$ Pain. 2009;13(5):439-40.

19. American Geriatrics Society Panel on the Pharmacological Management of Persistent Pain in Older Persons. Pharmacological management of persistent pain in older persons. J Am Geriatr Soc. 2009;57(8):1331-46.

20. Pickering G, Gibson SJ, Serbouti S, Odetti P, Ferraz Goncalves J, Gambassi G, et al. Reliability study in five languages of the translation of the pain behavioural scale Doloplus. Eur J Pain. 2010;14(5):545.e1-10.

21. Rat P, Jouve E, Pickering G, Donnarel L, Nguyen L, Michel M, et al. Validation of an acute pain-behavior scale for older persons with inability to communicate verbally: Algoplus. Eur J Pain. 2011;15(2):198.e1-10.

22. Wary B, Doloplus C. Doloplus-2, a scale for pain measurement. Soins Gerontol. 1999;19:25-7.

23. Tracy B, Sean Morrison R. Pain management in older adults. Clin Ther. 2013;35(11):1659-68.

24. Pickering G, Leplege A. Herpes zoster pain, postherpetic neuralgia, and quality of life in the elderly. Pain Pract. 2011;11(4):397-402.

25. Cohen SP, Mao J. Neuropathic pain: mechanisms and their clinical implications. BMJ. 2014;348:f7656.

26. Kress HG, Ahlbeck K, Aldington D, Alon E, Coaccioli S, Coluzzi $\mathrm{F}$, et al. Managing chronic pain in elderly patients requires a CHANGE of approach. Curr Med Res Opin. 2014;30(6):1153-64.

27. Hadjistavropoulos T, Herr K, Prkachin KM, Craig KD, Gibson SJ, Lukas A, et al. Pain assessment in elderly adults with dementia. Lancet Neurol. 2014;13(12):1216-27.
28. van der Steen JT, Sampson EL, Van den Block L, Lord K, Vankova H, Pautex S, et al. Tools to assess pain or lack of comfort in dementia: a content analysis. J Pain Symptom Manag. 2015;50(5):659-675.e3.

29. Bouhassira D, Attal N, Alchaar H, Boureau F, Brochet B, Bruxelle $\mathrm{J}$, et al. Comparison of pain syndromes associated with nervous or somatic lesions and development of a new neuropathic pain diagnostic questionnaire (DN4). Pain. 2005;114(1-2):29-36.

30. Bouhassira D, Attal N, Fermanian J, Alchaar H, Gautron M, Masquelier E, et al. Development and validation of the Neuropathic Pain Symptom Inventory. Pain. 2004;108(3):248-57.

31. Freynhagen R, Baron R, Gockel U, Tolle TR. painDETECT: a new screening questionnaire to identify neuropathic components in patients with back pain. Curr Med Res Opin. 2006;22(10):1911-20.

32. Galer BS, Jensen MP. Development and preliminary validation of a pain measure specific to neuropathic pain: the Neuropathic Pain Scale. Neurology. 1997;48(2):332-8.

33. Krause SJ, Backonja MM. Development of a neuropathic pain questionnaire. Clin J Pain. 2003;19(5):306-14.

34. Portenoy R. Development and testing of a neuropathic pain screening questionnaire: ID pain. Curr Med Res Opin. 2006;22(8):1555-65.

35. Backonja MM, Krause SJ. Neuropathic pain questionnaire-short form. Clin J Pain. 2003;19(5):315-6.

36. Johnston SS, Udall M, Alvir J, McMorrow D, Fowler R, Mullins D. Characteristics, treatment, and health care expenditures of Medicare supplemental-insured patients with painful diabetic peripheral neuropathy, post-herpetic neuralgia, or fibromyalgia. Pain Med. 2014;15(4):562-76.

37. Pickering G. Analgesic use in the older person. Curr Opin Support Palliat Care. 2012;6(2):207-12.

38. Pickering G. Antiepileptics for post-herpetic neuralgia in the elderly: current and future prospects. Drugs Aging. 2014;31(9):653-60.

39. Abdulla A, Adams N, Bone M, Elliott AM, Gaffin J, Jones D, et al. Guidance on the management of pain in older people. Age Ageing. 2013;42(Suppl 1):i1-57.

40. Makris UE, Abrams RC, Gurland B, Reid MC. Management of persistent pain in the older patient: a clinical review. JAMA. 2014;312(8):825-36.

41. Molton IR, Terrill AL. Overview of persistent pain in older adults. Am Psychol. 2014;69(2):197-207.

42. Hurst H, Bolton J. Assessing the clinical significance of change scores recorded on subjective outcome measures. J Manip Physiol Ther. 2004;27(1):26-35.

43. Finnerup NB, Attal N, Haroutounian S, McNicol E, Baron R, Dworkin RH, et al. Pharmacotherapy for neuropathic pain in adults: a systematic review and meta-analysis. Lancet Neurol. 2015;14(2):162-73.

44. Vranken JH. Elucidation of pathophysiology and treatment of neuropathic pain. Cent Nerv Syst Agents Med Chem. 2012;12(4):304-14.

45. Fine PG. Treatment guidelines for the pharmacological management of pain in older persons. Pain Med. 2012;13(Suppl 2):S57-66.

46. Katz B. The science and art of pain management in older persons: case study and discussion. Pain Med. 2012;13(Suppl 2):S72-8.

47. Haanpaa M, Cruccu G, Nurmikko TJ, McBride WT, Docu Axelarad A, Bosilkov A, et al. Capsaicin $8 \%$ patch versus oral pregabalin in patients with peripheral neuropathic pain. Eur $\mathbf{J}$ Pain. 2016;20(2):316-28. 